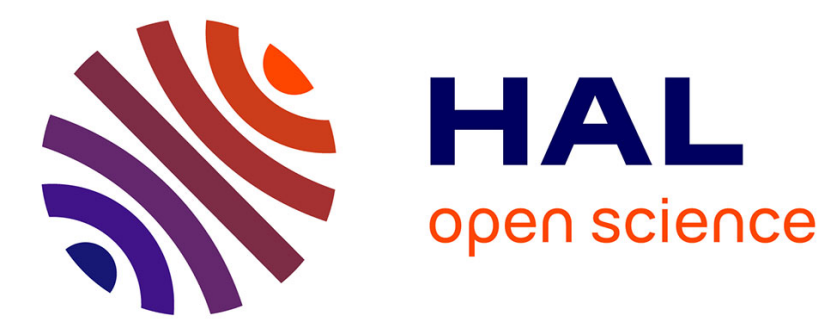

\title{
Photoacoustic imaging through a cortical bone replica with anisotropic elasticity
}

J Shepherd, Guillaume Renaud, P Clouzet, K. van Wijk

\section{To cite this version:}

J Shepherd, Guillaume Renaud, P Clouzet, K. van Wijk. Photoacoustic imaging through a cortical bone replica with anisotropic elasticity. Applied Physics Letters, 2020, 116, 10.1063/5.0011260 . hal-02879602v2

\section{HAL Id: hal-02879602 \\ https://hal.science/hal-02879602v2}

Submitted on 2 Jul 2020

HAL is a multi-disciplinary open access archive for the deposit and dissemination of scientific research documents, whether they are published or not. The documents may come from teaching and research institutions in France or abroad, or from public or private research centers.
L'archive ouverte pluridisciplinaire HAL, est destinée au dépôt et à la diffusion de documents scientifiques de niveau recherche, publiés ou non, émanant des établissements d'enseignement et de recherche français ou étrangers, des laboratoires publics ou privés. 


\title{
Photoacoustic imaging through a cortical bone replica with anisotropic elasticity
}

\author{
J. Shepherd, ${ }^{1, \text { a) }}$ G. Renaud, ${ }^{2, \text { b) }}$ P. Clouzet, ${ }^{2, c)}$ and K. van Wijk ${ }^{1, d)}$ \\ 1) Dodd-Walls Centre for Photonic and Quantum Technologies, Department of Physics, University of Auckland, Private Bag 92019 , \\ Auckland, New Zealand, 1010. \\ 2) Sorbonne Université, CNRS UMR 7371, INSERM UMR S 1146, Laboratoire d'Imagerie Biomédicale, Paris, \\ France
}

(Dated: 1 July 2020)

Photoacoustic (PA) imaging is an emerging modality which combines the high optical absorption contrast of biological chromophores with centimeter imaging depths and sub-millimeter resolution of ultrasonic (US) waves. However, PA imaging through cortical bone remains an unmet challenge. Cortical bone is an anisotropic medium which is not accurately modeled with existing PA image reconstruction methods. In this Letter, we address the PA source localization problem for imaging through a cortical bone-mimicking layer. Our approach accounts for both refraction and elastic anisotropy to accurately reconstruct an US and PA image in the presence of a cortical bone replica. We demonstrate our technique with a PA and US experiment, where we image a $700 \mu \mathrm{m}$ diameter target beneath a cortical bone-mimicking plate. Pulse-echo US is used to estimate the wavespeed in each layer and create an anatomical image of the bone replica, and the PA source is reconstructed in reception-only using the wavespeed model defined with US. We compute the thickness of the plate with less than $1 \%$ error, whereas isotropic assumptions overestimate the thickness by $20 \%$ or more. Incorporating both refraction and anisotropy accurately localizes the target with PA and US at the true depth, whereas isotropic assumptions blur the lateral dimension and mislocate the target depth by 1.5-4 mm.

Photoacoustic (PA) imaging is capable of generating highcontrast images of blood vessels non-invasively using the endogenous contrast of hemoglobin in the blood stream ${ }^{1,2}$. In reflection/epi-mode, a nanosecond pulse of diffuse light is absorbed by hemoglobin, creating a transient pressure rise which propagates as an ultrasonic "PA" wave to the tissue surface where it recorded by ultrasonic (US) detectors. Reconstructing the origin of the PA source creates images of optical absorption contrast with US resolution at multiple centimeter imaging depths.

However, mainstream PA reconstruction methods ${ }^{3-5}$ approximate all tissues as an isotropic, homogeneous fluid and cannot image beyond the first interface of bone (periosteum). The PA rays are assumed to travel in straight lines from the source (optical absorber) to receiver (US probe). This assumption is reasonable for soft-tissues, but breaks down in the presence of bone, which is an anisotropic material ${ }^{6-8}$ with a higher wavespeed than soft-tissues. In reality, PA rays refract upon crossing into and out of cortical bone, and experience an anisotropic wavespeed within cortical bone, as shown in Fig. 1.

Non-invasive detection of PA signals in the human brain has been shown ${ }^{9}$, demonstrating that sufficient light penetrates through a bone layer to generate measurable PA signals. However, PA imaging through a bone layer has not yet been shown. Recent work has successfully removed aberrations in numerical and experimental phantom data due to PA wave propagation through an isotropic skull model toward the goal

\footnotetext{
a)Electronic mail: jami.shepherd@auckland.ac.nz

(previously published as Jami L. Johnson)

b) Electronic mail: guillaume.renaud@upmc.fr

c)Electronic mail: pierre.clouzet@upmc.fr

d)Electronic mail: k.vanwijk@auckland.ac.nz
}

of transcranial PA imaging ${ }^{10,11}$. However, a known, isotropic wavespeed model is assumed and must be found with X-ray computed tomography $(\mathrm{CT})^{10}$. Isotropic assumptions based on CT scans are also used for transcranial US therapy ${ }^{12,13}$. While these examples focus on measurement, therapy, and imaging through the skull (cancellous bone), the challenges for imaging through cortical bone are related. In cortical bone, the preferential orientation of pores and mineralized fibrils result in an anisotropic wavespeed distribution in the longitudinal plane, where the wavespeed is more than $20 \%$ higher along the bone diaphysis (axial direction) than in the radial direction perpendicular to the diaphysis ${ }^{8}$. Therefore, isotropic wavespeed assumptions are insufficient for accurate imaging in the presence of cortical bone ${ }^{14}$.

In this Letter, we present a methodology which accounts for both refraction and elastic anisotropy to address the acoustic source localization problem for PA imaging beneath a cortical bone-mimicking layer with anisotropic elasticity. US data are acquired along with the PA data to measure the wavespeed model and create anatomical US images of the bone layer without the aid of an external modality, such as CT. US and PA data are routinely acquired concurrently with dual-modality systems ${ }^{15,16}$, and in human US imaging of the bone cortex has recently been demonstrated ${ }^{14,17}$.

The experimental setup is shown in Fig. 1. An $8 \mathrm{~mm}$ cortical bone-mimicking plate is suspended in a water bath above an optically absorbing target ( $700 \mu \mathrm{m}$ diameter graphite rod). The bottom of the rod is located at a depth of $23 \mathrm{~mm}$. The plate thickness is on the order of the thickness of the posterior cortex of a human femur ${ }^{18}$, where the effects of anisotropy and refraction would be especially pronounced. The bone plate (Sawbones, Pacific Research Laboratory Inc, Vashon WA) has been studied previously, and found to have similar elastic properties to human cortical bone ${ }^{19}$. In particular, both Sawbones and cortical bone are transverse-isotropic ${ }^{7}$. The 


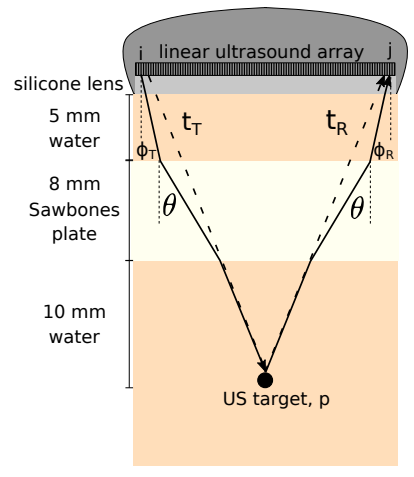

(a) pulse-echo ultrasound

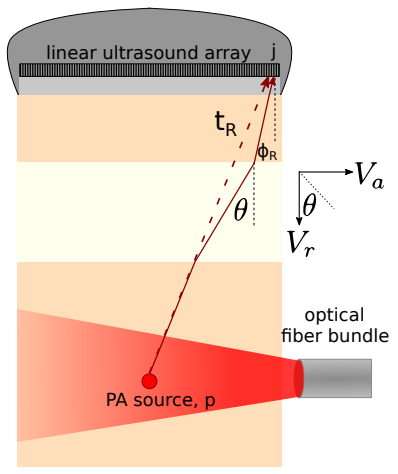

(b) photoacoustic

FIG. 1. Pulse-echo ultrasound (US) and photoacoustic (PA) imaging through a cortical bone-mimicking layer. An optically absorbing target is located beneath a cortical bone-mimicking plate (Sawbones). Conventional reconstruction methods assume the PA and US rays travel in straight lines (dotted) through a homogeneous, isotropic medium. However, cortical bone is an anisotropic material which causes rays to refract upon entering and exiting the cortical bone layer (solid lines).

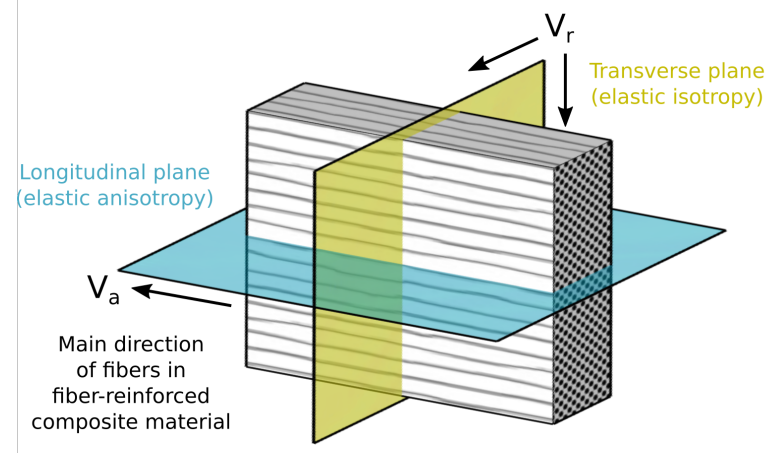

FIG. 2. The cortical bone-mimicking plate is transverse-isotropic: the wavespeed model is isotropic in the transverse plane, and anisotropic in the longitudinal plane. The symmetry axis is along the main direction of the fibers.

wavespeed is isotropic in the transverse plane perpendicular to the material fibril orientation, and anisotropic in the longitudinal plane parallel to the fibrils (Fig. 2).

Water is used to approximate the cutaneous tissue and bone marrow layers. Soft-tissue (acoustic) assumptions are good approximations for these tissues ${ }^{20}$, though the magnitude of the wavespeed in the cutaneous tissues and bone marrow will differ in $v i v o^{21}$. The plate is optically opaque, therefore we illuminate the PA target below the plate with a nanosecond pulsed laser coupled to an optical fiber bundle (OPOTEK Radiant $532 \mathrm{LD}$ ). The laser wavelength is $680 \mathrm{~nm}$ with a pulse width of $5 \mathrm{~ns}, 20 \mathrm{~Hz}$ repetition rate, and energy density $\sim 10 \mathrm{~mJ} \mathrm{~cm}^{-2}$. Optical illumination considerations for noninvasive in vivo PA imaging in bone are detailed at the end of the Letter.

Both US and PA data were acquired using a programmable US probe (L11-5v, 7.6 MHz center frequency) and an ultrafast

TABLE I. Elastic properties used for the image reconstruction.

\begin{tabular}{ccccc}
\hline \hline$V_{\text {lens }}$ & $V_{\text {water }}$ & $V_{a}$ & $V_{r}$ & $\beta$ \\
$1003 \mathrm{~ms}^{-1}$ & $1486 \mathrm{~m} \mathrm{~s}^{-1}$ & $3843 \mathrm{~m} \mathrm{~s}^{-1}$ & $3032 \mathrm{~m} \mathrm{~s}^{-1}$ & 1.95 \\
\hline \hline
\end{tabular}

US system (Verasonics, Vantage 128). In a preliminary experiment, we acquired data in both the longitudinal (anisotropic) and transverse (isotropic) plane to compute the wavespeed model in the bone plate. Each element was independently fired, and the resulting wavefield was recorded on all elements simultaneously to create an inter-element matrix. The axial wavespeed $V_{a}$, radial wavespeed $V_{r}$, and anisotropic form parameter $\beta$ of the bone plate are computed using the procedure detailed in Ref. 14, with one variation. To compute the headwave speed along the axial direction, we apply a linearmoveout correction ${ }^{22,23}$ in the time-domain rather than the frequency domain approach described in Ref. 14. All elastic properties are computed automatically given wavespeed bounds and depth parameters as inputs, and listed in Table I. For imaging, we acquired PA data as well as a complete interelement US matrix in the longitudinal plane. Mode-converted shear waves and surface-related multiple reflections are muted in the raw US data.

Delay-and-sum (DAS) beamforming for US imaging computes travel times between two points in a 2D medium with coordinates $\left(x_{1}, z_{1}\right)$ and $\left(x_{2}, z_{2}\right)$ with the distance equation:

$$
t=\frac{\sqrt{\left(x_{1}-x_{2}\right)^{2}+\left(z_{1}-z_{2}\right)^{2}}}{V},
$$

assuming a constant wavespeed $V$. In soft-tissues, $\left(x_{1}, z_{1}\right)$ is normally fixed as the coordinates of an array element and $\left(x_{2}, z_{2}\right)$ is the coordinates of an image pixel $p$.

The US image $\left(I_{U S}\right)$ is then computed

$$
I_{U S}(p)=\sum_{i=1}^{M} \sum_{j=1}^{N} W(p, i, j) \times D_{U S}\left[t=t_{T}(i, p)+t_{R}(j, p), i, j\right]
$$

where the image at each pixel $p$ is the summation of wavefield data $D_{U S}$ recorded at time $t$, for a single emitter $i$ and receiver $j$. The total travel time is the summation of transmit times $t_{T}$ for a ray travelling from $i$ to $p$, and receive times $t_{R}$ for a ray travel from $p$ to $j$ (Fig. 1). The one-way propagation time for a PA wave generated by a source at $p$ which propagates to $j$ is equivalent to $t_{R}$ computed in the US reconstruction. Therefore, a PA image is computed:

$$
I_{P A}(p)=\sum_{j=1}^{N} W(p, j) \times D_{P A}\left[t=t_{R}(j, p), j\right] .
$$

Each data point in $D_{U S}$ and $D_{P A}$ is multiplied by a weighting factor $W$ to reduce side lobe artifacts. Data outside of a predefined acceptance angle are multiplied by zero, and within the acceptance angle are multiplied by 1 .

Conventional DAS computes travel times assuming straight-ray paths through a homogeneous, isotropic medium. 


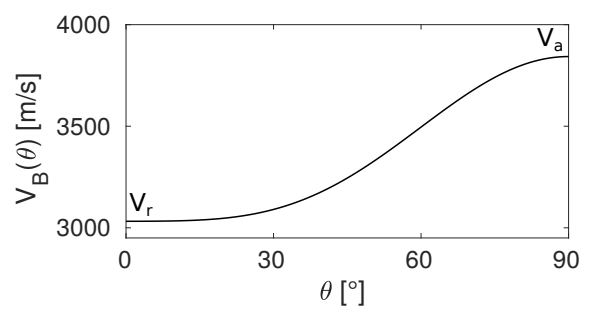

FIG. 3. Angular dependence of wavespeed within the bone layer corresponding to Eq. 4 and the properties in Table I.

For bone, the travel-times must be adjusted for the true propagation times through the higher-wavespeed, anisotropic cortical bone. To achieve this, we implement a modified DAS approach based on Kirchhoff migration ${ }^{24}$ which incorporates both refraction and elastic anisotropy.

First, we reconstruct an US image layer-by-layer ${ }^{14,17}$. For each layer, we compute both $t_{T}$ and $t_{R}$ using identical methods, therefore we discuss $t_{T}$ exclusively in the following.

An US image in the cutaneous tissue (water) layer is computed to a predefined depth. The travel times in the water layer are computed assuming both the silicone lens and water are isotropic, homogeneous fluids with wavespeeds listed in Table I. At the (known) lens-water interface, we apply Snell's law which refracts the ray according to the acoustic impedance and emerging angle $\phi_{T}$. With these travel times, the US image of the water layer is reconstructed using Eq. 2. The brightest reflector in this water layer is the first bone interface, which is segmented with the Dijkstra algorithm ${ }^{25,26}$ and fit to a parabola.

Within the cortical bone layer, refraction and anisotropy must be taken into account. Each ray begins at an emitter $i$, and Eq. 1 is first applied through the lens and tissue layer. When a ray reaches a pixel on the parabola corresponding to the water-bone interface, refraction is enforced according to the acoustic impedance between the water layer and bone, and the ray changes direction according to Snell's law from $\phi_{T}$ to $\theta$ as in Fig. 1. Within the bone layer, Eq. 1 is applied, but now $\left(x_{1}, z_{1}\right)$ corresponds to the point on the interface. Further, the anisotropic (angle-dependent) group wavepeed $V_{B}(\theta)$ replaces $V$. To compute $V_{B}(\theta)$, we use Thomsen's equation for weak anisotropy ${ }^{27}$ :

$$
V_{B}(\theta)=V_{a}-\left(V_{a}-V_{r}\right) \times\left[\beta \sin ^{2} \theta \cos ^{2} \theta+\cos ^{4} \theta\right],
$$

as shown in Fig. 3 using the properties listed in Table I. A range of $\phi_{T}$ are tested, until the minimum travel time from $i$ to $p$ within the bone layer is found. With these travel times incorporating refraction and anisotropy, Eq. 2 is used to compute the bone layer as in standard DAS.

Next, we segment and fit a parabola to the bone-water interface, the brightest reflector in the bone layer. For the marrow (water) layer, we follow an identical procedure to the bone layer, but this time following a ray from $i$, through the first interface and bone layer, and enforce refraction a second time at the bone-water interface. Within the marrow layer, an isotropic wavespeed $V_{\text {water }}$ is used. Finally, after computation of $t_{T}$ and $t_{R}$ in the US reconstruction, we reconstruct the PA image with Eq. 3.

$\mathrm{PA}$ and US imaging results using conventional DAS and our bone imaging approach assuming both an isotropic and anisotropic wavespeed in bone are shown in Fig. 4. The US image is Hilbert transformed and log compressed for display. All non-positive values in the PA image are thresholded to zero $^{28}$, and the PA image is normalized and overlayed onto the US image in red.

The lateral and depth dimension across the peak corresponding to the top of the target measured with PA and US, as well as the thickness of the bone layer computed with US are reported in Table II. Generated PA and scattered US signals are observed from both the top and bottom of the target, along with reverberations. Therefore the dimensions are measured for the signal from the top of the target only. The dimensions are measured by normalizing the marrow image (without log compression) and extracting perpendicular cross sections across the peak corresponding to the top of the target in the image. Cross-sections are upsampled by a factor of 10 , and the width of the peak at $-10 \mathrm{~dB}$ is measured.

In Fig. 4(a), a DAS algorithm assuming a homogeneous, isotropic medium $\left(V=V_{\text {water }}\right)$ is used. The target is unfocused and the depth is largely underestimated with both PA and US. The interfaces of the bone plate are also improperly mapped, and the thickness is underestimated by $\sim 29 \%$. In Fig. 4(b), we utilise our refraction-based approach with an isotropic wavespeed model for the bone plate, where the optimum wavespeed $\left(3677 \mathrm{~m} \mathrm{~s}^{-1}\right)$ in bone is chosen by optimising the image quality ${ }^{29}$. The thickness of the plate is overestimated by $\sim 20 \%$. While the target is better focused in Fig. 4(b) than Fig. 4(a), it is located at the wrong depth and the lateral diameter is blurred in both PA and US images. In Fig 4(c), the true thickness of the plate is found to be $7.96 \mathrm{~mm}$, an error of less $\ll 1 \%$ when compared to caliper measurements $(7.97 \pm$ $0.02 \mathrm{~mm})$. The lateral dimension at $-10 \mathrm{~dB}$ is smallest when utilising the anisotropic model for both PA and US and the target is located within $100 \mu \mathrm{m}$ of the true depth.

In our phantom experiment, the angle of optical illumination is such that the light avoids propagation through the opaque bone-mimicking replica. For in vivo PA imaging, optical transmission through soft-tissue and bone will be hampered by intrinsic losses, but recording PA signals through a bone layer has already proven feasible in vivo ${ }^{9}$. Consider a model with $5 \mathrm{~mm}$ of cutaneous soft-tissue and a cortical bone thickness ranging from $\sim 4 \mathrm{~mm}$ typical of a human radius, to the thicker cortex of the human femur $(\sim 8 \mathrm{~mm})$. The effective optical attenuation coefficient $\mu_{\text {eff }}$ in soft-tissue within the optical window is ${ }^{1} \sim 0.13 \mathrm{~mm}^{-1}$, therefore, the initial fluence will reduce by $\sim 50 \%$ through the soft-tissue layer. In bone, we approximate ${ }^{30} \mu_{\text {eff }} \sim 0.17 \mathrm{~mm}^{-1}$, reducing the fluence by another $50 \%$ through a $4 \mathrm{~mm}$ bone layer and $75 \%$ through an $8 \mathrm{~mm}$ layer. For an initial fluence of $20 \mathrm{~mJ} / \mathrm{cm}^{2}$, the fluence remaining in the bone marrow of the radius will be $\sim 5 \mathrm{~mJ} / \mathrm{cm}^{2}$ and in the femur $\sim 2.5 \mathrm{~mJ} / \mathrm{cm}^{2}$, sufficient for measurable PA generation. Accounting for loss-of-energy due to reflection of the US and PA waves, optical and acoustic attenuation, we estimate that PA imaging beneath $5 \mathrm{~mm}$ of soft- 


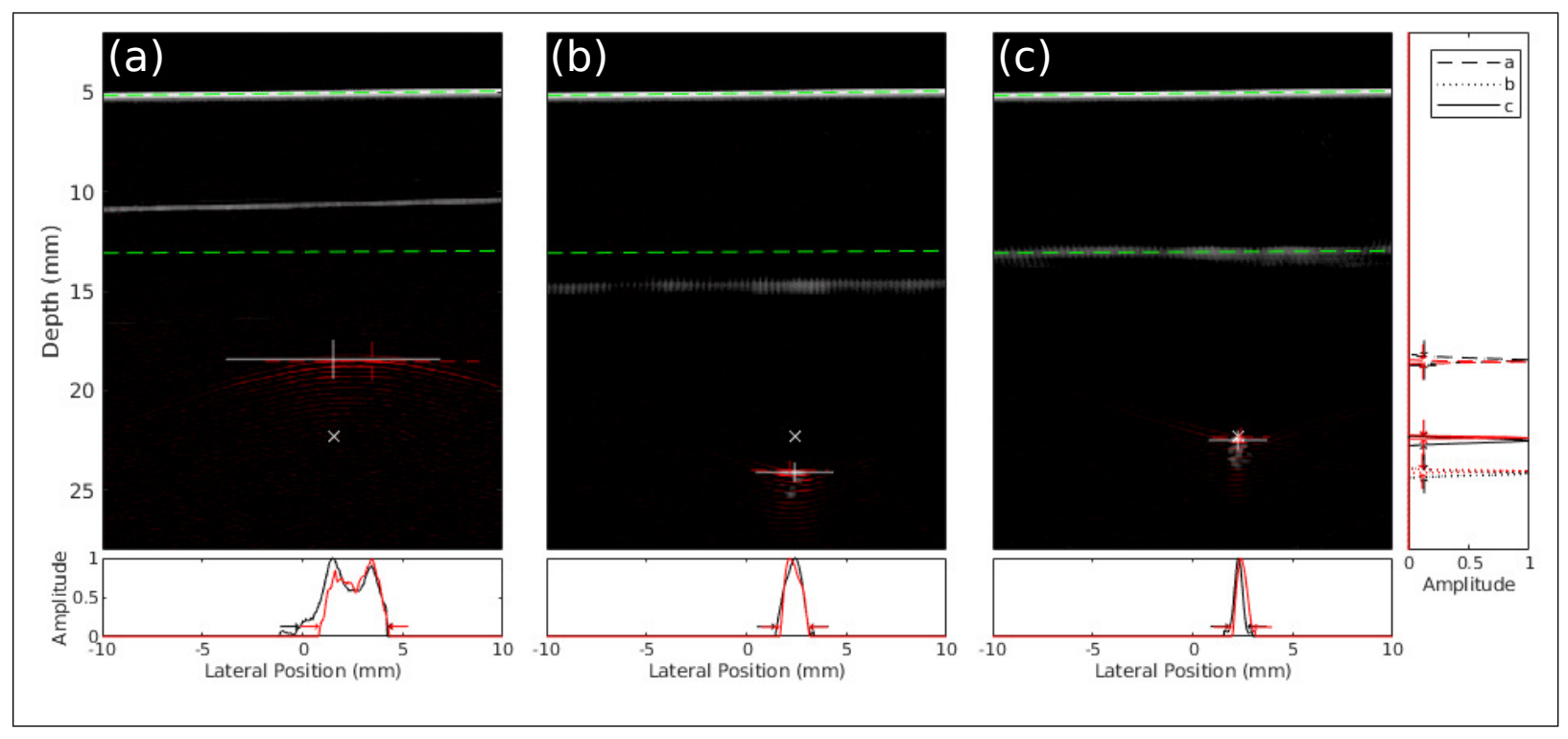

FIG. 4. US (grayscale) and PA (red overlay) images reconstructed using (a) DAS assuming a constant wavespeed of $V_{\text {water }}=1486 \mathrm{~ms} \mathrm{~s}^{-1}$ and refraction-corrected imaging with a (b) layered medium including a bone layer with isotropic elasticity and (c) layered medium including a bone layer with anisotropic elasticity. The interfaces segmented in the US image using the method in (c) are shown with a dotted green line on all three panels. The true location of the top of the target is marked ' $X$ '. Cross-sections along the lateral and depth direction are extracted at the point in the PA (dotted red lines) and US (white lines) images corresponding to the top of the target. The PA and US cross sections are shown in red and black, respectively. The width at $-10 \mathrm{~dB}$ of each cross section is denoted with arrows.

TABLE II. US and PA imaging results with different assumptions about the acousto-elastic properties of the medium.

\begin{tabular}{c|ccc}
\hline \hline wavespeed model & $\begin{array}{c}\text { uniform medium: } \\
\text { "soft-tissue" }\end{array}$ & $\begin{array}{c}\text { layered medium: } \\
\text { bone layer, isotropic elasticity }\end{array}$ & $\begin{array}{c}\text { layered medium: } \\
\text { bone layer, anisotropic elasticity }\end{array}$ \\
\hline thickness cortical bone replica & $5.67 \mathrm{~mm}$ & $9.62 \mathrm{~mm}$ & $7.96 \mathrm{~mm}$ \\
PA target depth (top) & $18.5 \mathrm{~mm}$ & $23.9 \mathrm{~mm}$ & $22.2 \mathrm{~mm}$ \\
US target depth (top) & $18.2 \mathrm{~mm}$ & $23.9 \mathrm{~mm}$ & $22.3 \mathrm{~mm}$ \\
PA dimensions (lateral, depth) & $3383 \mu \mathrm{m}, 156 \mu \mathrm{m}$ & $1354 \mu \mathrm{m}, 176 \mu \mathrm{m}$ & $924 \mu \mathrm{m}, 161 \mu \mathrm{m}$ \\
US dimensions (lateral, depth) & $4331 \mu \mathrm{m}, 386 \mu \mathrm{m}$ & $1589 \mu \mathrm{m}, 396 \mu \mathrm{m}$ & $797 \mu \mathrm{m}, 416 \mu \mathrm{m}$ \\
\hline \hline
\end{tabular}

tissue and $4 \mathrm{~mm}$ of cortical bone (i.e. the bone marrow of the radius) with state-of-the-art probes even up to $10 \mathrm{MHz}$ is feasible, but lower probe frequencies $\lesssim 7 \mathrm{MHz}$ will be required to image through bones with a thicker cortex, such as the femur. Therefore, we conclude that PA images of blood perfusion through the cortex of superficial long bones in reflection/epi mode are feasible. Nonetheless, we highlight that even pessimistic PA imaging depths of several millimeters through cortical bone will provide valuable information about bone perfusion with a point-of-care modality. The optical absorption coefficient for hemoglobin ${ }^{1}$ is substantially higher than bone ${ }^{30}$, thus we expect the PA blood signal in bone to be high contrast in vivo.

Multiple reflections and mode-converted waves are weak in this example. However, due to the high impedance mismatch between soft-tissues and cortical bone, reflection artifacts will become more prominent when PA waves are generated in the cutaneous tissue and cortical bone layers. Several approaches are considered to suppress these artifacts. Waveequation based image reconstruction such as time reversal $(\mathrm{TR})^{31,32}$ could be modified to incorporate anisotropy, handle mode-converted waves, and correct for acoustic attenuation ${ }^{33}$. TR has been applied to soft-tissue models ${ }^{34}$, and we have demonstrated that these propagators can be applied to both PA (TR) and US (reverse-time migration) ${ }^{16}$ data. Further, TR has shown to reduce reflection artifacts in numerical models with multiple wavespeeds ${ }^{35}$. Alternatively, pre-processing of the data can eliminate multiple reflections before reconstruction using Marchenko imaging ${ }^{36}$ or PAFUSion ${ }^{37}$.

In this Letter, we provide a solution to the acoustic source localization challenge for PA imaging through a cortical bonemimicking layer using only US and PA data. Further, we confirm that both refraction and elastic anisotropy must be incorporated for accurate PA imaging through a cortical bonemimicking layer, and isotropic assumptions result in significant errors in the image reconstruction.

The authors thank the Photon Factory at the University of Auckland for the Verasonics ultrasound system and the DoddWalls Centre for Photonic and Quantum Technologies for ongoing support. The data supporting the findings of this study are available upon reasonable request. 


\section{REFERENCES}

${ }^{1}$ P. Beard, “Biomedical photoacoustic imaging," Interface Focus 1, 602-631 (2011).

${ }^{2}$ M. Xu and L. V. Wang, "Photoacoustic imaging in biomedicine," Review of Scientific Instruments 77, 041101 (2006)

${ }^{3} \mathrm{M}$. Xu and L. V. Wang, "Universal back-projection algorithm for photoacoustic computed tomography," Physical Review E 71, 016706 (2005).

${ }^{4}$ P. Burgholzer, G. J. Matt, M. Haltmeier, and G. Paltauf, "Exact and approximative imaging methods for photoacoustic tomography using an arbitrary detection surface," Physical Review E 75, 046706 (2007).

${ }^{5}$ B. E. Treeby and B. T. Cox, "k-wave: Matlab toolbox for the simulation and reconstruction of photoacoustic wave fields," Journal of Biomedical Optics 15, 021314 (2010).

${ }^{6}$ X. Cai, L. Peralta, P.-J. Gouttenoire, C. Olivier, F. Peyrin, P. Laugier, and Q. Grimal, "Quantification of stiffness measurement errors in resonant ultrasound spectroscopy of human cortical bone," The Journal of the Acoustical Society of America 142, 2755-2765 (2017).

${ }^{7}$ S. Bernard, J. Schneider, P. Varga, P. Laugier, K. Raum, and Q. Grimal, "Elasticity-density and viscoelasticity-density relationships at the tibia mid-diaphysis assessed from resonant ultrasound spectroscopy measurements," Biomechanics and Modeling in Mechanobiology 15, 97-109 (2016).

${ }^{8}$ M. Granke, Q. Grimal, A. Saied, P. Nauleau, F. Peyrin, and P. Laugier, "Change in porosity is the major determinant of the variation of cortical bone elasticity at the millimeter scale in aged women," Bone 49, 10201026 (2011)

${ }^{9}$ S. Yamamoto, C. Robertson, Y. Petrov, I. Petrov, R. Esenaliev, and D. Prough, "Continuous, noninvasive optoacoustic monitoring of cerebral venous blood oxygenation in patients with traumatic brain injury," Journal of Neurotrama 34, A62-A62 (2017).

${ }^{10}$ C. Huang, L. Nie, R. W. Schoonover, Z. Guo, C. O. Schirra, M. A. Anastasio, and L. V. Wang, "Aberration correction for transcranial photoacoustic tomography of primates employing adjunct image data," Journal of Biomedical Optics 17, 066016 (2012).

${ }^{11}$ K. Mitsuhashi, J. Poudel, T. P. Matthews, A. Garcia-Uribe, L. V. Wang, and M. A. Anastasio, "A forward-adjoint operator pair based on the elastic wave equation for use in transcranial photoacoustic computed tomography," SIAM Journal on Imaging Sciences 10, 2022-2048 (2017).

${ }^{12}$ J.-F. Aubry, M. Tanter, M. Pernot, J.-L. Thomas, and M. Fink, "Experimental demonstration of noninvasive transskull adaptive focusing based on prior computed tomography scans," The Journal of the Acoustical Society of America 113, 84-93 (2003).

${ }^{13}$ F. Marquet, M. Pernot, J.-F. Aubry, G. Montaldo, L. Marsac, M. Tanter, and M. Fink, "Non-invasive transcranial ultrasound therapy based on a $3 \mathrm{~d}$ ct scan: protocol validation and in vitro results," Physics in Medicine \& Biology 54, 2597-2614 (2009).

${ }^{14}$ G. Renaud, P. Kruizinga, D. Cassereau, and P. Laugier, "In vivo ultrasound imaging of the bone cortex," Physics in Medicine and Biology 63, 125010 (2018).

${ }^{15}$ M. K. A. Singh, W. Steenbergen, and S. Manohar, "Handheld probe-based dual mode ultrasound/photoacoustics for biomedical imaging," in Frontiers in Biophotonics for Translational Medicine (Springer, 2016) pp. 209-247.

${ }^{16}$ J. L. Johnson, J. Shragge, and K. van Wijk, "Nonconfocal all-optical laserultrasound and photoacoustic imaging system for angle-dependent deep tissue imaging," Journal of Biomedical Optics 22, 041014 (2017).

${ }^{17}$ G. Renaud, J. Johnson, and D. Cassereau, "Real-time kirchhoff migration for ultrasound imaging of the bone cortex," in SEG Technical Program Expanded Abstracts 2018 (2018) pp. 4797-4801.
${ }^{18}$ S. L. Croker, W. Reed, and D. Donlon, "Comparative cortical bone thickness between the long bones of humans and five common non-human mammal taxa," Forensic Science International 260, 104-e1 (2016).

${ }^{19}$ S. Bernard, Q. Grimal, and P. Laugier, "Resonant ultrasound spectroscopy for viscoelastic characterization of anisotropic attenuative solid materials," The Journal of the Acoustical Society of America 135, 2601-2613 (2014).

${ }^{20}$ P. Laugier and G. Haïat, "Introduction to the physics of ultrasound," in Bone Quantitative Ultrasound (Springer, 2011) pp. 29-45.

${ }^{21} \mathrm{P}$. Nicholson and M. Bouxsein, "Effect of temperature on ultrasonic properties of the calcaneus in situ," Osteoporosis International 13, 888-892 (2002).

${ }^{22}$ W. M. Telford, W. Telford, L. Geldart, R. E. Sheriff, and R. Sheriff, Applied Geophysics (Cambridge University Press, 1990).

${ }^{23}$ J. L. Johnson, K. van Wijk, and M. Sabick, "Characterizing phantom arteries with multi-channel laser ultrasonics and photo-acoustics," Ultrasound in Medicine \& Biology 40, 513-520 (2014)

${ }^{24}$ J. Etgen, S. H. Gray, and Y. Zhang, "An overview of depth imaging in exploration geophysics," Geophysics 74, WCA5-WCA17 (2009).

${ }^{25} \mathrm{E}$. W. Dijkstra, "A note on two problems in connexion with graphs," Numerische Mathematik 1, 269-271 (1959).

${ }^{26} \mathrm{D}$. Hong, "Medical image segmentation based on accelerated dijkstra algorithm," in Advances in Intelligent Systems (Springer, 2012) pp. 341-348.

${ }^{27}$ L. Thomsen, "Weak elastic anisotropy," Geophysics 51, 1954-1966 (1986).

${ }^{28}$ B. E. Treeby, J. Jaros, and B. T. Cox, "Advanced photoacoustic image reconstruction using the k-wave toolbox," in Photons Plus Ultrasound: Imaging and Sensing 2016, Vol. 9708 (International Society for Optics and Photonics, 2016) p. $97082 \mathrm{P}$

${ }^{29}$ B. E. Treeby, T. K. Varslot, E. Z. Zhang, J. G. Laufer, and P. C. Beard, "Automatic sound speed selection in photoacoustic image reconstruction using an autofocus approach," Journal of Biomedical Optics 16, 090501 (2011).

${ }^{30}$ J. L. Sandell and T. C. Zhu, "A review of in-vivo optical properties of human tissues and its impact on pdt," Journal of Biophotonics 4, 773-787 (2011).

${ }^{31} \mathrm{Y}$. Xu and L. V. Wang, "Time reversal and its application to tomography with diffracting sources," Physical review letters 92, 033902 (2004).

${ }^{32}$ Y. Hristova, P. Kuchment, and L. Nguyen, "Reconstruction and time reversal in thermoacoustic tomography in acoustically homogeneous and inhomogeneous media," Inverse Problems 24, 055006 (2008).

${ }^{33}$ B. E. Treeby, E. Z. Zhang, and B. T. Cox, "Photoacoustic tomography in absorbing acoustic media using time reversal," Inverse Problems 26, 115003 (2010).

${ }^{34}$ G. Paltauf, R. Nuster, M. Haltmeier, and P. Burgholzer, "Photoacoustic tomography using a mach-zehnder interferometer as an acoustic line detector," Applied optics 46, 3352-3358 (2007).

${ }^{35}$ H. Grün, G. Paltauf, M. Haltmeier, and P. Burgholzer, "Photoacoustic tomography using a fiber based fabry-perot interferometer as an integrating line detector and image reconstruction by model-based time reversal method," in European Conference on Biomedical Optics (Optical Society of America, 2007) p. 6631_6.

${ }^{36}$ J. van der Neut, J. L. Johnson, K. van Wijk, S. Singh, E. Slob, and K. Wapenaar, "A marchenko equation for acoustic inverse source problems," The Journal of the Acoustical Society of America 141, 4332-4346 (2017).

${ }^{37}$ M. K. A. Singh, M. Jaeger, M. Frenz, and W. Steenbergen, "In vivo demonstration of reflection artifact reduction in photoacoustic imaging using synthetic aperture photoacoustic-guided focused ultrasound (pafusion)," Biomedical optics express 7, 2955-2972 (2016). 